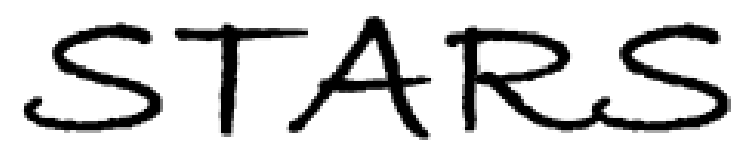

University of Central Florida

STARS

$1-1-2006$

\title{
Terahertz amplification in delta-doped germanium films with in- plane transport
}

\author{
M. V. Dolguikh \\ University of Central Florida
}

A. V. Muravjov

University of Central Florida

R. E. Peale

University of Central Florida

Find similar works at: https://stars.library.ucf.edu/facultybib2000

University of Central Florida Libraries http://library.ucf.edu

This Article is brought to you for free and open access by the Faculty Bibliography at STARS. It has been accepted for inclusion in Faculty Bibliography 2000 s by an authorized administrator of STARS. For more information, please contact STARS@ucf.edu.

\section{Recommended Citation}

Dolguikh, M. V.; Muravjov, A. V.; and Peale, R. E., "Terahertz amplification in delta-doped germanium films with in-plane transport" (2006). Faculty Bibliography 2000s. 6088.

https://stars.library.ucf.edu/facultybib2000/6088

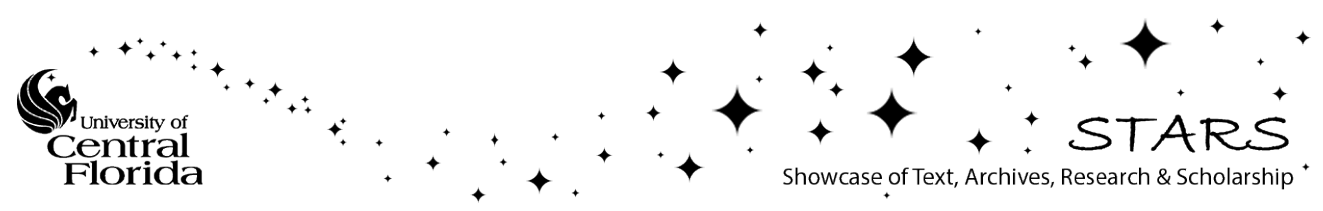




\section{Terahertz amplification in delta-doped germanium films with in-plane transport}

Cite as: J. Appl. Phys. 99, 093106 (2006); https://doi.org/10.1063/1.2193067

Submitted: 13 October 2005. Accepted: 09 March 2006 . Published Online: 12 May 2006

M. V. Dolguikh, A. V. Muravjov, and R. E. Peale

\section{Lock-in Amplifiers up to $600 \mathrm{MHz}$}

starting at

$\$ 6,210$

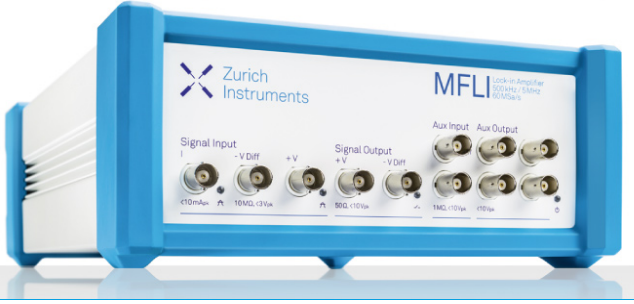

J. Appl. Phys. 99, 093106 (2006); https://doi.org/10.1063/1.2193067

(c) 2006 American Institute of Physics. 


\title{
Terahertz amplification in delta-doped germanium films with in-plane transport
}

\author{
M. V. Dolguikh, A. V. Muravjov, and R. E. Peale ${ }^{a)}$ \\ Department of Physics, University of Central Florida, Orlando, Florida 32816 and College of Optics \\ and Photonics, University of Central Florida, Orlando, Florida 32816
}

(Received 13 October 2005; accepted 9 March 2006; published online 12 May 2006)

\begin{abstract}
Amplification of terahertz radiation on intersubband transitions has been analyzed by numerical Monte Carlo simulation for $p$-type delta-doped Ge films with in-plane transport configuration of applied electric and magnetic fields. A significant increase of the gain is found, compared to existing bulk $p$-Ge lasers, due to spatial separation of light and heavy hole streams, which reduces scattering of light holes on ionized impurities and heavy holes. The considered device has potential as a widely tunable $(2-4 \mathrm{THz})$ laser with high duty cycle and operating temperatures up to $50 \mathrm{~K}$. (C) 2006 American Institute of Physics. [DOI: 10.1063/1.2193067]
\end{abstract}

\section{INTRODUCTION}

Generation of terahertz stimulated emission on direct optical transitions between valence subbands for bulk $p$-Ge crystals in crossed electric $E$ and magnetic $B$ fields is well known. ${ }^{1,2}$ The lifetime of magnetized light holes, which defines the population inversion, depends on three major scattering processes. Acoustic phonon scattering, which increases with lattice temperature, tends to thermalize the desired nonequilibrium hole distribution. Scattering of light holes on ionized impurities limits the maximum acceptable concentration of acceptors, and thus the concentration of active carriers, to the level of $\sim 10^{14} \mathrm{~cm}^{-3}$, so that the gain has not exceeded $0.1-0.2 \mathrm{~cm}^{-1}$ in homogeneously doped $p$-Ge lasers. Carrier-carrier collisions also cause intersubband and intrasubband transitions. ${ }^{3}$ We recently presented ${ }^{4}$ a planar $p$-Ge laser concept with vertical transport, in which periodic delta doping reduces scattering of light holes on impurities and heavy holes, thus increasing the upper laser-level lifetime. The resulting increase in gain would allow operating temperatures up to $77 \mathrm{~K}$ and continuous duty cycle while preserving the feature in bulk $p$-Ge lasers of broad tunability $(2-4 \mathrm{THz})$. In this paper we present a different configuration of the intersubband terahertz laser on delta-doped multilayer Ge thin films, in which the transport is predominantly in plane. Spatial separation of light and heavy hole streams is achieved, but with significantly larger carrier concentrations than possible for vertical transport, which allows much higher gain. Theoretical results for the considered device obtained by means of Monte Carlo simulation are presented here.

A practical goal is development of a terahertz laser based on thin germanium films with small signal gain coefficient sufficient to operate at liquid nitrogen temperatures. An additional motivating factor for pursuing this approach, when terahertz quantum cascade lasers have already achieved this temperature milestone, ${ }^{5,6}$ is the unique very wide gain spectrum $(2-4 \mathrm{THz})$ for the transitions between valence subbands. This feature is potentially important for building a

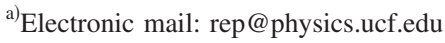

terahertz laser with wide tunability, which none of the other known terahertz semiconductor lasers possesses. ${ }^{7}$

\section{THEORETICAL METHODS}

Hole dynamics, hole distribution functions, and the gain on direct optical light-to-heavy hole transitions are calculated by the Monte Carlo simulation method using classical motion equations and hole scattering probabilities. ${ }^{8,9}$ Two valence subbands (light and heavy holes) with isotropic and parabolic dispersion laws are considered. The isotropic approach is justified by the small warping of the germanium valence band. We can neglect quantum confinement effects because the considered structure has no heteroboundaries, only delta-doped layers. We also neglect Landau quantization, which is a good approximation for magnetic fields of $2 \mathrm{~T}$ and below. Time or ensemble averaged momentum and position yield the hole distribution functions $f_{l, h}(\mathbf{k}, \mathbf{r})$ [subband $(l, h)$, wave vector $\mathbf{k}$, and coordinate $\mathbf{r}]$. The distribution functions were considered uniform in the horizontal planes according to the geometry of the problem. The standard Rees rejection technique chooses among scattering processes. ${ }^{8}$ The rate of each scattering process is given by a temperature-dependent analytic expression. Optical phonon scattering is treated in a deformation potential approximation. ${ }^{10,11}$ Acoustic phonon scattering is simplified according to Ref. 12. Inelasticity for acoustic phonon scattering is included. ${ }^{13}$ Brooks-Herring model ${ }^{14}$ with inverse Debye screening length and Yukawa potential was used for ionized impurity scattering. Hole-hole scattering was calculated according to Ref. 3. Iteration determines the self-consistent solution of the Poisson equation and thereof the spatial carrier distribution and potential profile. ${ }^{4}$ The small signal gain is calculated as the difference between the gain on direct intersubband (light-to-heavy hole) transitions and free carrier absorption assisted by phonons and ionized impurities. ${ }^{13,15} \mathrm{Up}$ to liquid nitrogen temperatures and impurity concentrations of $\sim 10^{15}-10^{16} \mathrm{~cm}^{-3}$, the main contribution to free carrier absorption comes from optical phonon emission by heavy holes. ${ }^{15}$ Lattice absorption ${ }^{16}$ was not included in the calculations and was considered separately (see below). 


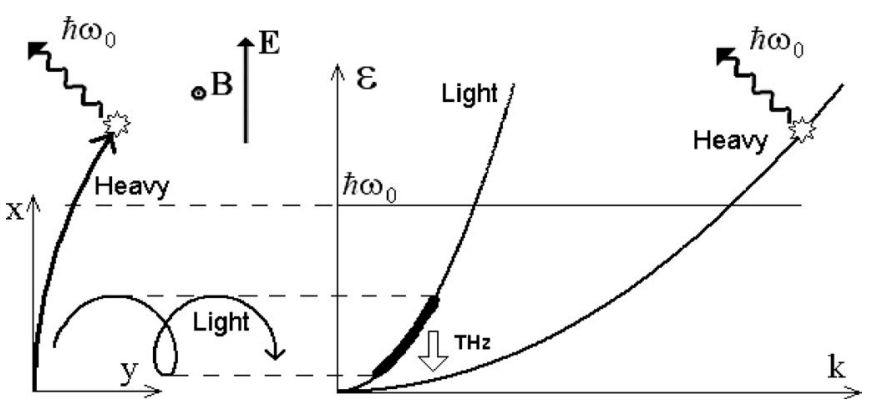

FIG. 1. Mechanism of stimulated emission on direct optical transitions between light and heavy hole subbands. The left graph shows light and heavy hole orbits in the plane perpendicular to the applied magnetic field. The right figure shows the allowed energies of light and heavy holes as a function of the magnitude of the momentum wave vector. Heavy holes scatter rapidly with optical phonon emission. Light holes are magnetized, have much smaller scattering rates, and therefore have a long lifetime. Overpopulation of the light hole subband results in terahertz gain on direct optical light-toheavy hole transitions.

\section{CALCULATION RESULTS}

The mechanism of building up the population inversion in $p$-type germanium in crossed electric and magnetic fields is presented in Fig. 1. Due to different effective masses, the ratio between the magnitudes of applied electric and magnetic fields can be chosen (typically $E=1.5 \mathrm{kV} / \mathrm{cm}$ and $B$ $=1 \mathrm{~T}$ ), so that light holes become confined on cyclotron trajectories below the optical phonon energy threshold, giving them long lifetimes. For the same fields, heavy hole trajectories are only slightly perturbed, allowing frequent scattering on optical phonons.

The in-plane carrier transport configuration for active delta-doped germanium structures is presented in Fig. 2. The magnetic field is oriented along the layers. The orientation of the electric field is chosen to keep the heavy hole current $J_{h}$

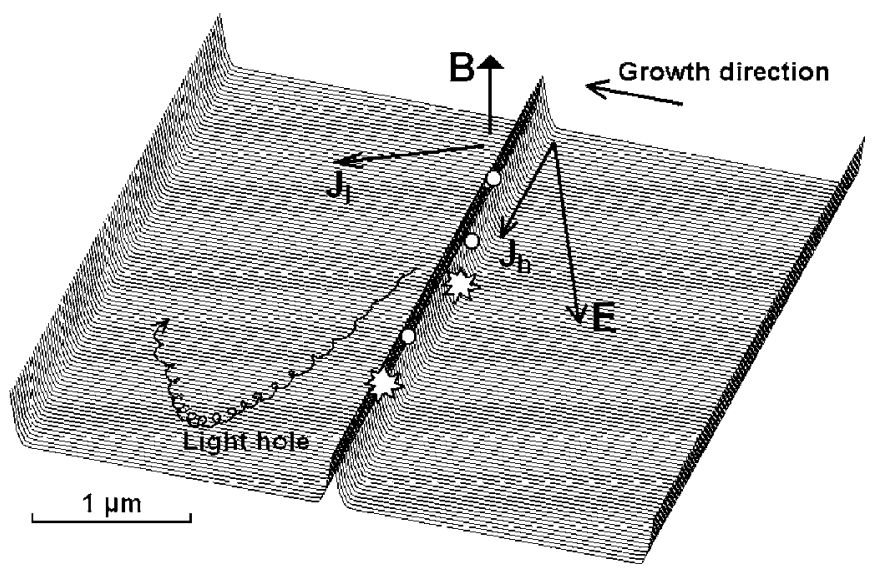

FIG. 2. Schematic of in-plane transport of carriers in periodically deltadoped $p$-type germanium structures in crossed electric and magnetic fields. The surface indicates the periodic doping profile. Currents $J_{h}$ and $J_{l}$ and electric field $\mathbf{E}$ are coplanar and perpendicular to the applied $\mathbf{B}$ field. $\mathbf{E}$ is the superposition of the applied electric field and the Hall field. Electric $(2-3 \mathrm{kV} / \mathrm{cm})$ and magnetic $(\sim 2 \mathrm{~T})$ fields, applied as shown, provide strong current of heavy holes $J_{h}$ along heavily doped layers with intense ionized impurity and phonon scattering (indicated by open circles and stars, respectively). Light holes are pulled from the layers by the fields and have component of current $J_{l}$ across the layers. These light holes have a long lifetime in the undoped regions, with their trajectories defined by the self-consistent built-in electric field.

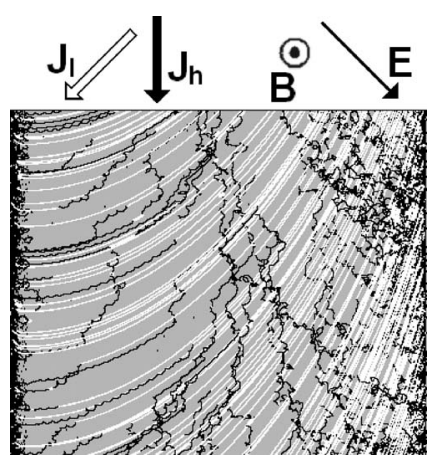

FIG. 3. Monte Carlo simulated hole trajectories within one period of the delta-doped structure in crossed electric and magnetic fields. $E=3 \mathrm{kV} / \mathrm{cm}$, $B=2 \mathrm{~T}$, period $d=2000 \mathrm{~nm}$, and average hole concentration is 1.5 $\times 10^{15} \mathrm{~cm}^{-3}$. The white trajectories represent light holes, while the black trajectories represent heavy holes.

within the doped layers. Light holes move across the undoped layers due to the drift in the direction perpendicular to both applied electric and magnetic fields. The structure period is chosen to roughly equal the average light hole free path at $10-20 \mathrm{~K}$ for the applied fields. The average carrier concentration is chosen to be $(1-2) \times 10^{15} \mathrm{~cm}^{-3}$. This transport configuration achieves spatial separation of light holes from both impurity and heavy holes. Though some heavy holes remain in the undoped regions their negative effect on the inversion population is less severe than that of ionized impurity centers. ${ }^{4}$

Figure 3 presents the pattern of hole trajectories within one period of the structure from the actual Monte Carlo simulation with parameters given in the caption. Heavy holes are depicted by thin black lines, which tend to flow downward along, and be concentrated within, the doped layers. Light holes (thin white lines) drift across the undoped region. On this scale, the cyclotron orbits of heavy holes are just visible while those of light holes are smaller than the width of the drift lines.

The calculated electric potential surface is presented in Fig. 4. In the given applied fields (Figs. 2 and 3) and in the presence of optical phonon scattering, heavy holes experience streaming motion along the doped layers. Because light holes are free from inelastic optical phonon scattering, they more rapidly gain a significant vertical component of $\mathbf{E} \times \mathbf{B}$

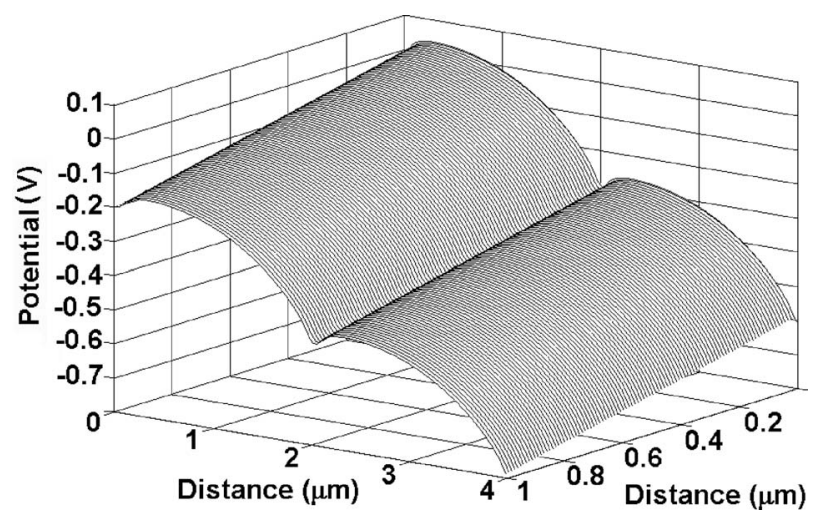

FIG. 4. Self-consistent potential profile calculated for the same parameters as Fig. 3, except $E=2.5 \mathrm{kV} / \mathrm{cm}$. 


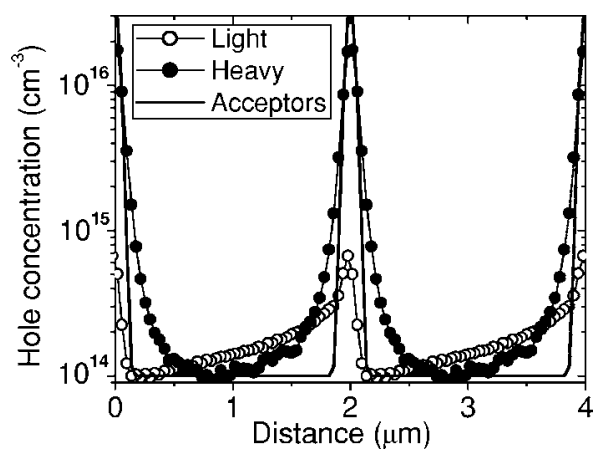

FIG. 5. Distribution of light and heavy hole concentrations across the structure. Most of the heavy holes are concentrated in the vicinity of the doped layers, whereas light holes have much higher concentration in the undoped regions than have heavy holes.

drift velocity. Thus, light holes enter the undoped regions in higher absolute numbers than heavy holes do.

Resulting light and heavy hole concentration distributions are shown in Fig. 5 together with the doping profile for a structure with average carrier concentration of 1.5 $\times 10^{15} \mathrm{~cm}^{-3}$ and $d=2000 \mathrm{~nm}$. In the center of the undoped regions, the concentration of light holes exceeds that of heavy holes. Considering the much higher density of states in the heavy hole band, this then represents a very highly inverted population in such regions of the crystal. The heavy holes moving along the doped layers create strong negative inversion in these regions. The concentration peak of light holes inside the doped layers represents light holes that experience transition to the heavy hole band before they have the chance of escaping to the undoped regions. These light holes partially compensate the negative inversion inside the doped layers created by the heavy holes.

Figure 6 presents the spatial-spectral gain distribution calculated with the same parameters as Figs. 4 and 5. Gain is shown as a function of optical transition wave number and vertical distance in the structures. Heavy black contours indicate the intersection of the gain surface with the zero-gain level. The gain surface has strong broad amplification bands in the undoped regions and narrow but strong absorption regions near the doped layers. The gain surface follows the carrier distribution in Fig. 5. Free carrier absorption has been included in Fig. 6.

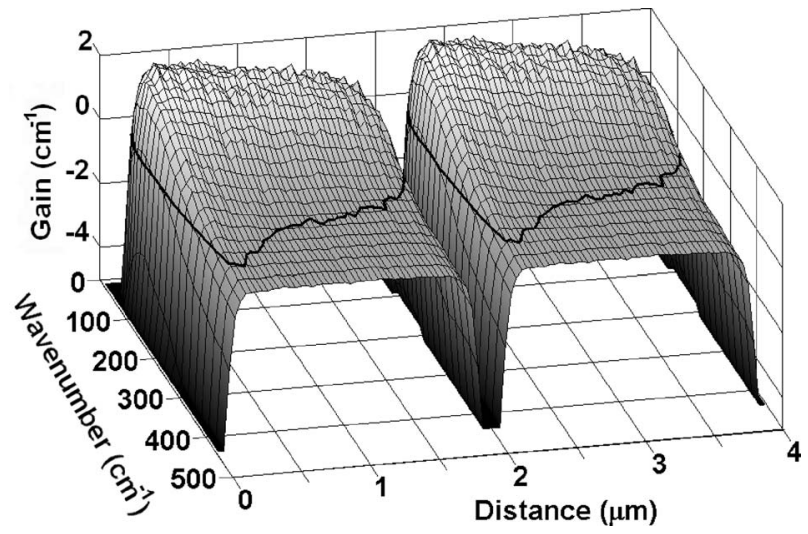

FIG. 6. Spatial-spectral gain distribution across two structure $\left(N_{\mathrm{av}}=1.5\right.$ $\left.\times 10^{15} \mathrm{~cm}^{-3}, d=2000 \mathrm{~nm}, E=2.5 \mathrm{kV} / \mathrm{cm}, B=2 \mathrm{~T}, T=10 \mathrm{~K}\right)$ with free carrier absorption included.

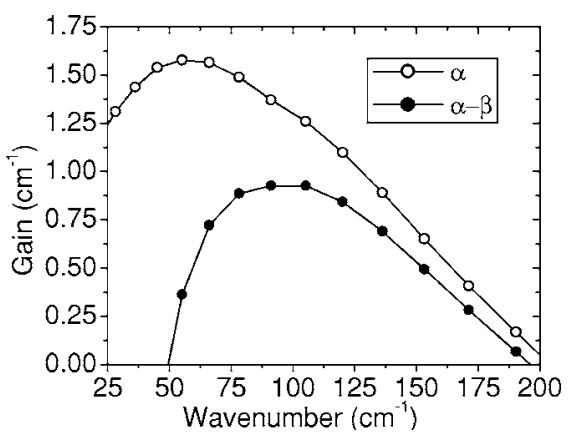

FIG. 7. Calculated spatially averaged terahertz gain $\alpha$ in the structure with parameters as for Figs. $4-6$. The net curve $\alpha-\beta$ includes the free carrier absorption coefficient $\beta$.

The spatially averaged net gain in the structure is presented in Fig. 7. Results with and without free carrier absorption are presented. Even with free carrier absorption, the gain at $100 \mathrm{~cm}^{-1}$ of nearly $1 \mathrm{~cm}^{-1}$ exceeds that in usual bulk $p$-Ge lasers by a factor of about 10 .

Figure 8 presents the dependence of the gain at $100 \mu \mathrm{m}$ wavelength on lattice temperature for two different structure periods. Comparison of the gain is made with experimental germanium lattice absorption at the same wavelength. ${ }^{16} \mathrm{Net}$ gain persists up to at least $40 \mathrm{~K}$. Calculations of the gain for different applied fields and structure periods confirm the expectation that the optimal period of the structure for the maximum average gain should approximately equal the mean free path of the light holes. For parameters as in Figs. $3-5$ this length is about $2 \mu \mathrm{m}$, which can be seen in Fig. 5 from the exponential decrease of the light hole concentration with the vertical distance from the doped layers. A smaller period would allow light holes to cross the undoped region and scatter on impurities in the next layer. Moreover, a smaller period allows relatively more frequent interaction between light and heavy holes. A larger period would increase the relative thickness of the absorbing regions near the doped layers. At higher temperatures (and higher doping concentrations), the mean free path decreases. This decreases the optimal period of the structure, which can be seen in Fig. 8 as the crossing of the 1 and $2 \mu \mathrm{m}$ curves near $40 \mathrm{~K}$.

\section{DISCUSSION AND CONCLUSION}

Means of creating an inversion population on direct optical transitions between light and heavy hole subbands with

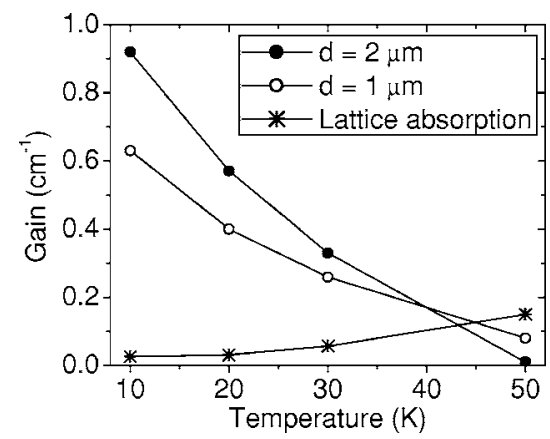

FIG. 8. Spatially averaged gain at $100 \mu \mathrm{m}$ wavelength as a function of lattice temperature. The legend indicates the structure period. Experimental germanium lattice absorption (Ref. 16) is presented for comparison. 
in-plane transport in delta-doped structures have been proposed theoretically and demonstrated with Monte Carlo simulation. The spatial separation of light holes from ionized impurities and separation of currents for light and heavy holes eliminate light hole scattering on ionized impurities and on the majority of heavy holes. This allows an increase of light hole concentration to the level of (1-3) $\times 10^{14} \mathrm{~cm}^{-3}$, which is ten times higher than the typical light hole concentration in bulk $p$-Ge lasers.

The desired separation of light and heavy hole streams requires the majority of heavy holes to flow along the doped layers, while light hole current has a vertical component. As shown in Figs. 2 and 3, this requires that the internal electric field have a vertical component in the growth direction. In case of trivial contact configuration on the edges of the structure, when net carrier transport is parallel to the doped layers, heavy hole current would point slightly into the undoped material, in order to compensate the vertical component of light hole current (see Fig. 2). This in turn could lead to smaller gain values than calculated. In order to achieve the optimal gain one should arrange a slight drain of the current in the vertical direction (approximately $10 \%$ of the in-plane current value) to adjust the direction of heavy hole current to lie within the doped layers. This can be achieved, in principle, by making an array of auxiliary contacts on the top and the bottom of the structure.

Due to the high gain estimates found for the considered device, higher temperature operation is possible than for bulk $p$-Ge lasers. The smaller active volume and planar geometry facilitate heat extraction, which will allow higher duty. The simplicity and low growth precision requirements allow chemical vapor deposition (CVD), which can produce structures of remarkable active thickness. High quality structures, similar to those discussed here, with the thickness above $30 \mu \mathrm{m}$ have been demonstrated. ${ }^{4}$ Due to the homoepitaxial nature of the structure and an absence of hetero interfaces, growth of $100-150 \mu \mathrm{m}$ thick structures without remarkable defect concentrations appears feasible. Such structures would be suitable for making low-loss confocal cavities for the spectral range of $2-4 \mathrm{THz}$.

\section{ACKNOWLEDGMENTS}

This work was partially supported by AFOSR Contract Nos. F49620-02-C-0025 and F49620-02-C-0027. One of the authors (R.E.P.) acknowledges support from an ASEE/AFRL summer faculty fellowship in 2005 and the encouragement of his host, Dr. Richard Soref.

\footnotetext{
${ }^{1}$ V. N. Shastin, Opt. Quantum Electron. 23, S111 (1991).

${ }^{2}$ E. Bründermann, in Long Wavelength Infrared Semiconductor Lasers, edited by H. K. Choi (Wiley, New Jersey, 2004), pp. 279-343.

${ }^{3}$ M. V. Dolguikh, A. V. Muravjov, and R. E. Peale, Phys. Rev. B 73, 075327 (2006).

${ }^{4}$ M. V. Dolguikh, A. V. Muravjov, R. E. Peale, M. Klimov, O. A. Kuznetsov, and E. A. Uskova, J. Appl. Phys. 98, 023107 (2005).

${ }^{5}$ B. S. Williams, H. Callebaut, S. Kumar, Q. Hu, and J. L. Reno, Appl. Phys. Lett. 82, 1015 (2003).

${ }^{6}$ B. S. Williams, S. Kumar, H. Callebaut, Q. Hu, and J. L. Reno, Appl. Phys. Lett. 83, 5142 (2003).

${ }^{7}$ A. V. Muravjov, M. V. Dolguikh, R. E. Peale, O. A. Kuznetsov, and E. A. Uskova, Proc. SPIE 5727, 44 (2005).

${ }^{8}$ C. Jacoboni and L. Reggiani, Rev. Mod. Phys. 55, 645 (1983).

${ }^{9}$ C. Jacoboni, R. Brunetti, and P. Bordone, in Theory of Transport Properties of Semiconductor Nanostructures, edited by E. Scholl (Chapman and Hall, London, 1998), p. 59.

${ }^{10}$ G. L. Bir and G. E. Pikus, Symmetry and Strain-induced Effects in Semiconductors (Wiley, New York, 1974).

${ }^{11}$ P. Lawaetz, Phys. Rev. 174, 867 (1968).

${ }^{12}$ J. D. Wiley, Phys. Rev. B 4, 2485 (1970).

${ }^{13}$ E. V. Starikov and P. N. Shiktorov, Opt. Quantum Electron. 23, S177 (1991).

${ }^{14}$ B. K. Ridley, Quantum Processes in Semiconductors (Oxford University Press, New York, 1999).

${ }^{15}$ Yu. K. Pozhela, E. V. Starikov, and P. N. Shiktorov, Phys. Status Solidi B 128, 653 (1985).

${ }^{16}$ R. Brazis and F. Keilmann, Solid State Commun. 70, 1109 (1989).
} 